\title{
SPATIAL CHARACTERISTICS OF F/A-18 VERTICAL TAIL BUFFET PRESSURES MEASURED IN FLIGHT
}

\author{
Robert W. Moses, AIAA Member \\ Aeroelasticity Branch \\ NASA Langley Research Center \\ Hampton, VA
}

\author{
Gautam H. Shah, AIAA Senior Member \\ Vehicle Dynamics Branch \\ NASA Langley Research Center \\ Hampton, VA
}

\begin{abstract}
$\underline{\text { Abstract }}$
Buffeting is an aeroelastic phenomenon which plagues high performance aircraft, especially those with twin vertical tails, at high angles of attack. Previous wind-tunnel and flight tests were conducted to characterize the buffet loads on the vertical tails by measuring surface pressures, bending moments, and accelerations. Following these tests, buffeting estimates were computed using the measured buffet pressures and compared to the measured responses. The estimates did not match the measured data because the assumed spatial correlation of the buffet pressures was not correct. A better understanding of the partial (spatial) correlation of the differential buffet pressures on the tail was necessary to improve the buffeting estimates. Several wind-tunnel investigations were conducted for this purpose. When combined and compared, the results of these tests show that the partial correlation depends on and scales with flight conditions. One of the remaining questions is whether the windtunnel data is consistent with flight data. Presented herein, cross-spectra and coherence functions calculated from pressures that were measured on the high alpha research vehicle (HARV) indicate that the partial correlation of the buffet pressures in flight agrees with the partial correlation observed in the wind tunnel.
\end{abstract}

Copyright (C) 1998 by the American Institute of Aeronautics and Astronautics, Inc. No copyright is asserted in the United States under Title 17, U. S. Code. The U. S. Government has a royalty-free license to exercise all rights under the copyright claimed herein for Governmental Purposes. All other rights are reserved by the copyright owner.

\section{$\underline{\text { Introduction }}$}

For high performance aircraft at high angles of attack, vortices emanating from wing/fuselage leading edge extensions (LEX) often burst, immersing the vertical tails in their wake, as shown in Figure 1 for the F/A-18. The resulting buffet loads on the vertical tails are a concern from airframe fatigue and maintenance points of view. Previous wind-tunnel and flight tests have been conducted to quantify the buffet loads on the vertical tails of the F/A-18.

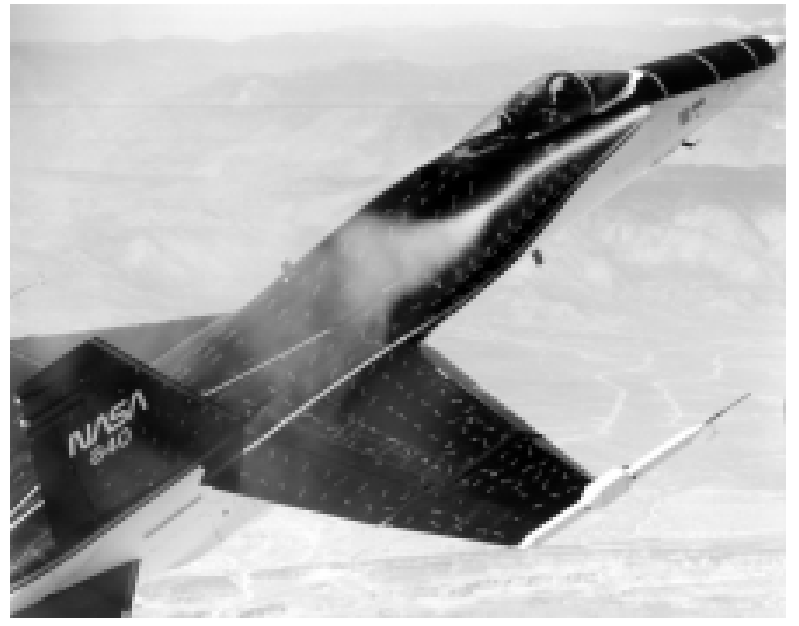

Figure 1. Flow Visualization of Leading Edge Extension (LEX) Vortex Burst, 30 Degrees Angle of Attack

The spectral nature of the unsteady differential pressures on the F/A-18 vertical tail caused by a burst LEX vortex are well documented' ${ }^{1}$ As illustrated in Reference 1, the power spectral densities and root mean square (rms) values of the differential pressures vary with flight speed, angle of attack, dynamic pressure, and tail position. The 
worst case buffet condition, defined by the highest rms values of differential pressure at design limit load, occurs on the F/A-18 aircraft around 340 pounds per square foot (psf) and 32 degrees angle of attack. Other findings of Reference 1 were that the root mean square value of the differential pressure varies linearly with dynamic pressure, and that Strouhal (proportional to reduced frequency) scaling provides a means for comparing model and flight data. Also, the highest rms values occur at stations closest to the leading edge while the lowest rms values occur near the trailing edge with a gradual change in rms values between these two regions of the tail. Another conclusion from this investigation was that the unsteady differential pressures were considered fully correlated (in phase) since the pressures measured at five stations did not indicate a significant phase shift.

After the research of Reference 1, wind-tunnel tests of a $6 \%$ rigid F/A-18 model were conducted to investigate the spatial characteristics of the unsteady surface pressures on the tail ${ }^{2}$. Contour plots of the time delays of the unsteady pressures on each surface at Mach 0.6 were constructed using cross-correlation analyses of the measured unsteady pressures. As shown in Figure 2 for 35 degrees angle of attack, the contours for inboard and outboard tail surfaces are quite different. Therefore, the spatial characteristics of the unsteady differential pressures are unclear.

Because of the perceived complexity in transcribing partially-correlated unsteady pressures into the analyses of buffet and buffeting, the differential pressures on the tail have been assumed to be in phase (fully correlated) at any given time ${ }^{3-5}$. These analyses do not estimate the buffet loads accurately, and it was concluded that the issue of pressure correlation is the key to successful buffet prediction and should be the subject of more research ${ }^{4-5}$.

To learn more about the pressure correlation, a full-scale F/A-18 was tested at high angles of attack at a maximum speed of $M=0.15$. Plots of the magnitudes and phase delays of the unsteady differential pressures at two streamwise stations were constructed using cross-spectral analyses of the unsteady pressures measured on each tail surface at two different angles of attack ${ }^{6-7}$. These two angles of attack of 20 degrees and 32 degrees, shown in Figure $3 a$ and $3 b$, represent a harmless and one of the worst buffet conditions for the F/A18. As shown in Figure $3 \mathrm{a}$ and $3 \mathrm{~b}$, the phase steadily decreases with increasing frequency. This trend is consistent for both angles of attack. Although flight conditions were not matched, the results of this wind-tunnel test indicate that the differential pressures acting on the tail are not in phase and therefore are not fully correlated. However, the relationships of flight conditions on pressure correlation is not clearly understood from these results.
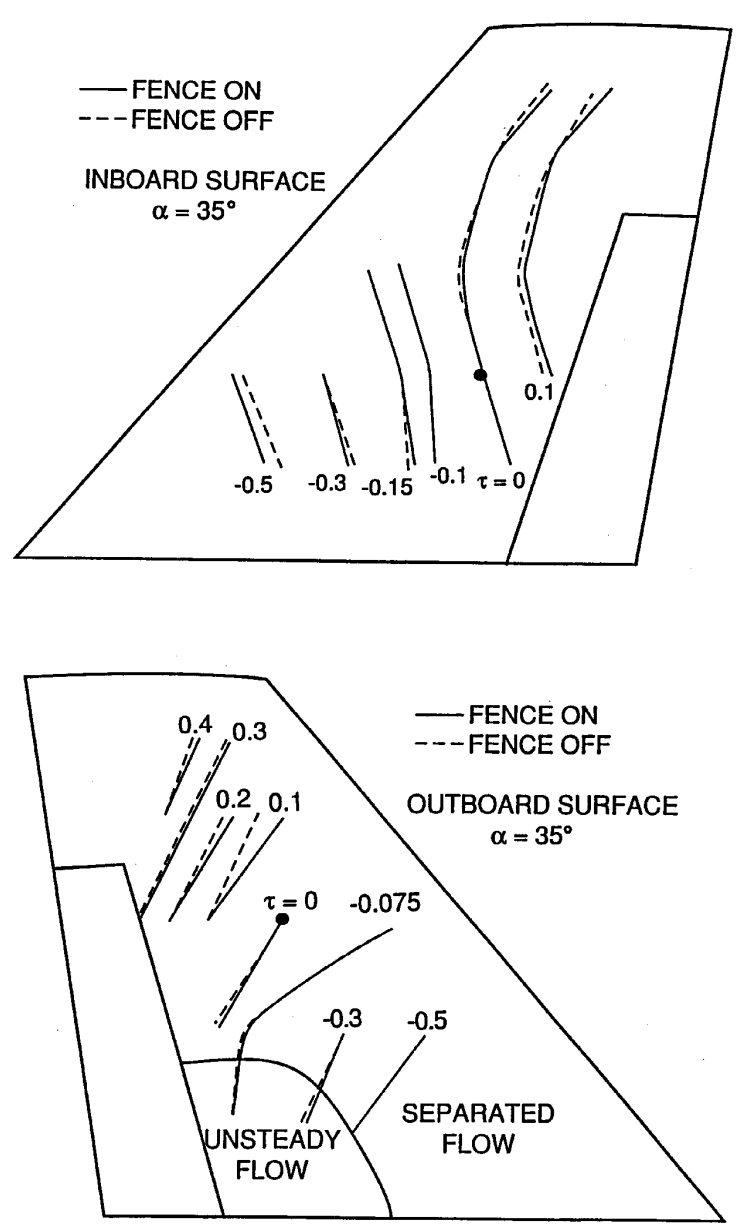

Figure 2. Peak Correlation Contours (msec) of the Fin Unsteady Pressure Signals, 6\% Rigid Tail, $\mathrm{M}=0.6,35$ Degrees AOA (From Reference 2) 


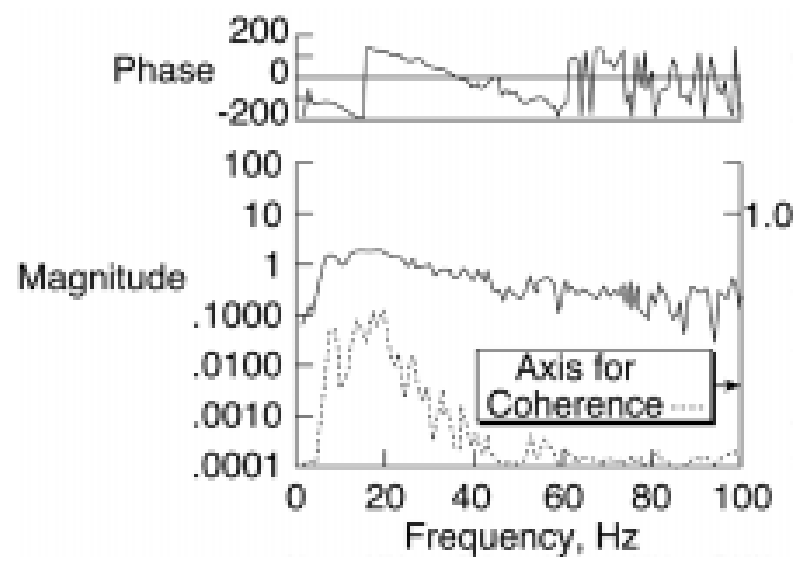

a) 20 Degrees $\mathrm{AOA}$

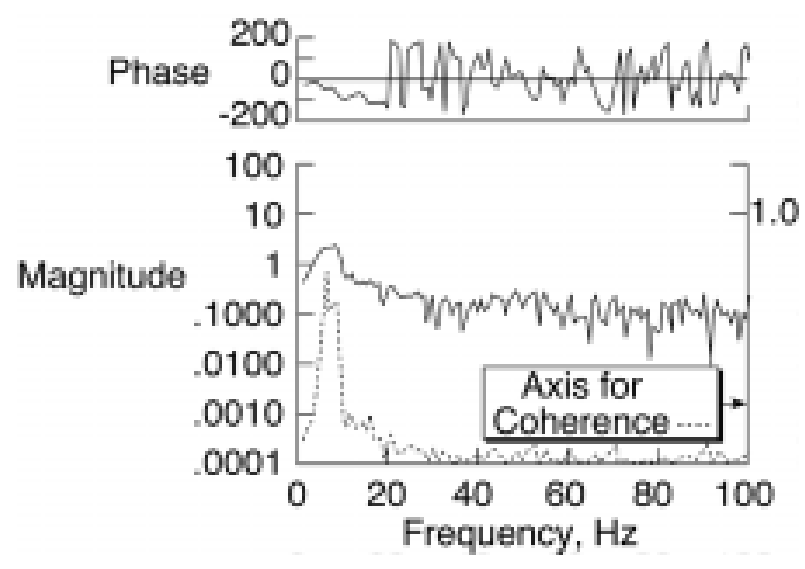

b) 32 Degrees AOA

Figure 3. Cross-Spectral Density and Coherence Functions Between the Differential Pressures Near the Leading-Edge Tip and the Trailing-Edge Tip,

Full-Scale Tail, M=0.15, (From Reference 6)

To better understand the pressure correlations during buffet, an available $16 \%$, sting-mounted, F18 wind-tunnel model, shown in Figure 4, was modified and tested in the Transonic Dynamics Tunnel (TDT) at the NASA Langley Research Center as part of the ACROBAT (Actively Controlled Response Of Buffet-Affected Tails) program $^{8}$. Surface pressures were measured for scaled flight conditions at high angles of attack on rigid and flexible tails (flexible tail is shown in Figure 5). Cross-correlation and cross-spectral analyses $^{9}$ were performed for identifying any consistent spatial characteristics of the unsteady differential pressures.

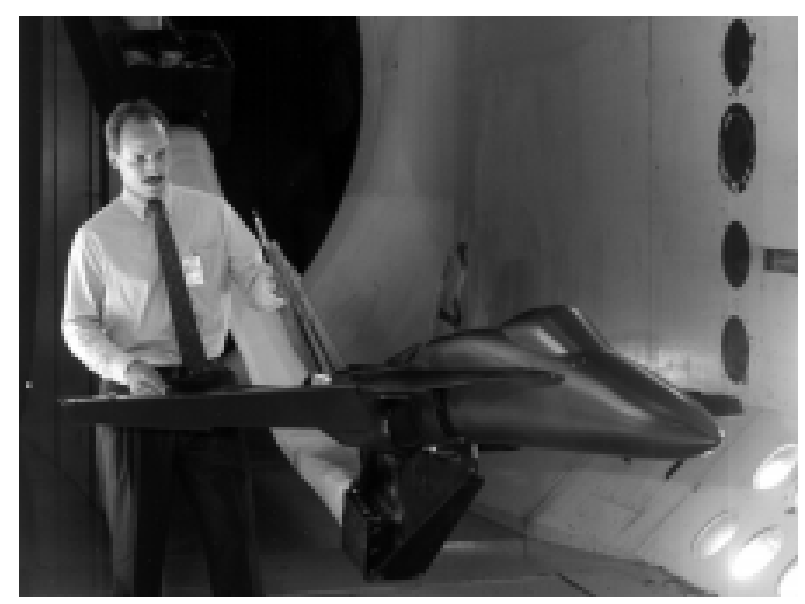

Figure 4. $16 \%$ F/A-18 Model Mounted in the Transonic Dynamics Tunnel

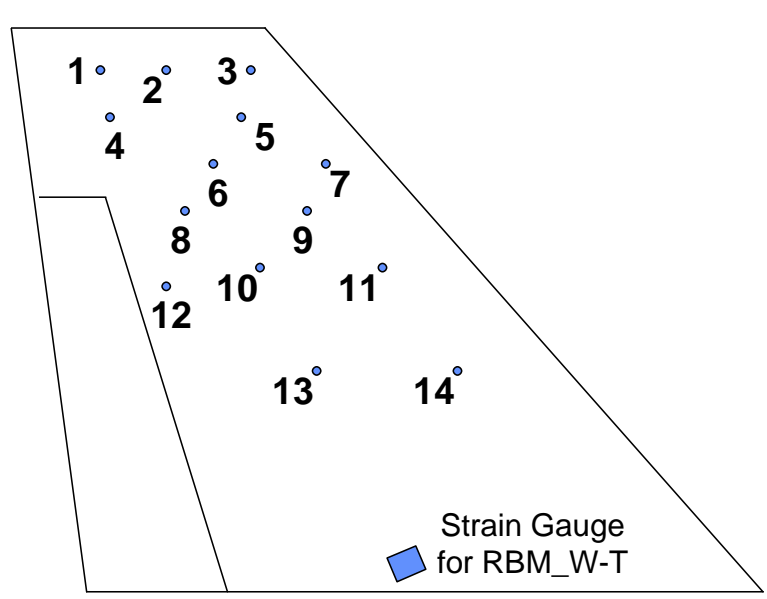

Figure 5. Pressure Transducer Stations, 1/6-Scale Flexible Tail

Cross-correlation and cross-spectral density functions of the unsteady differential pressures on the flexible tail were computed, as shown in Figures 6 and 7, respectively, and compared to results of References 2 and 6. These comparisons show that the time and phase delays of the unsteady differential pressures scale with windtunnel speed (and with reduced frequency) ${ }^{10}$. In fact, the unsteady differential pressures were found to resemble waves that move along the tail $^{11}$. 

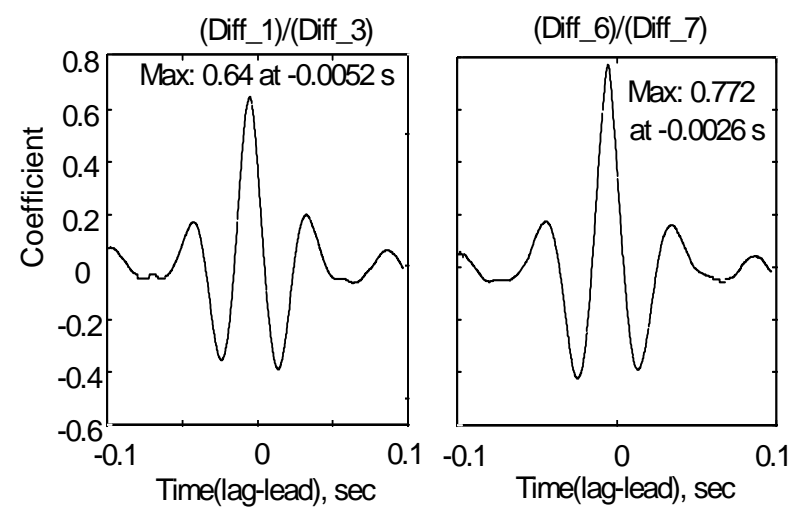

a) Near Tip and $75 \%$ Span
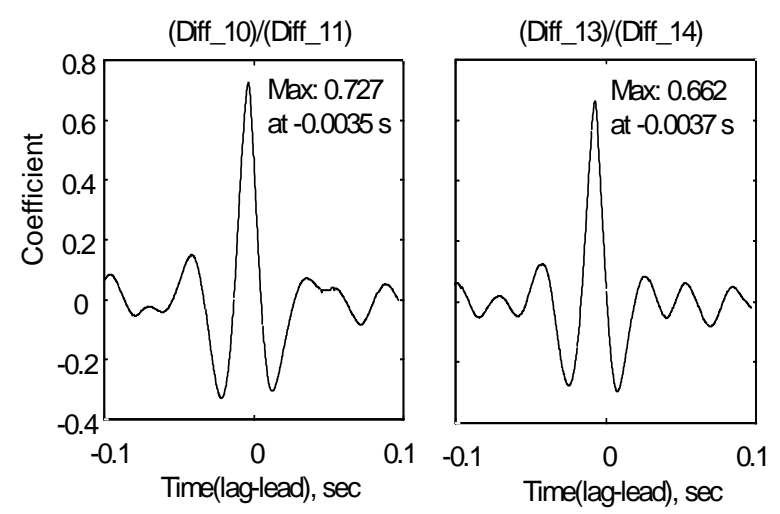

b) Near $60 \%$ and $40 \%$ Span

Figure 6. Cross-Correlation Functions Between Differential Pressures at Stations on Flexible Tail, 16\% F/A-18 Model, 34 Degrees AOA
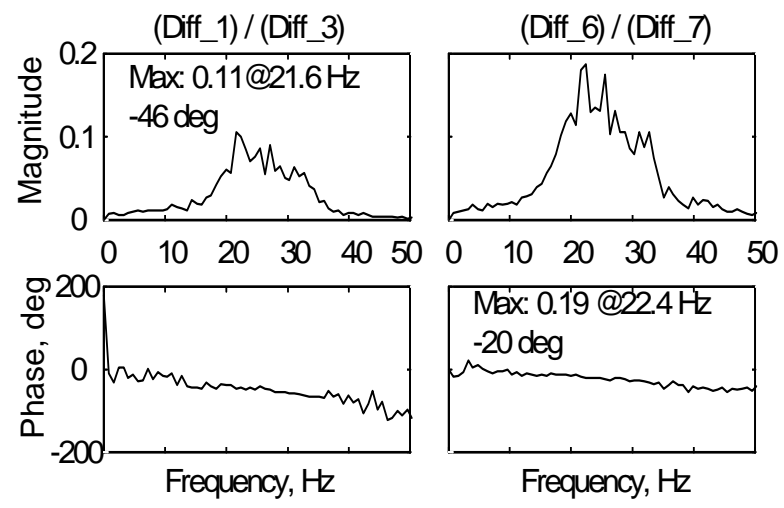

a) Near Tip and $75 \%$ Span

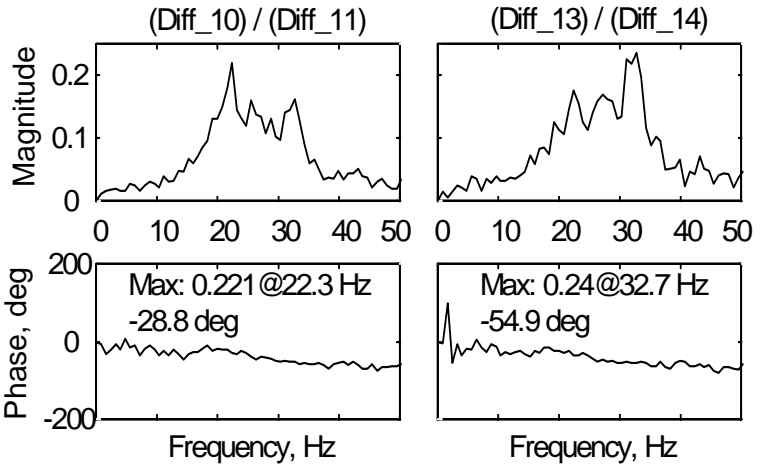

b) Near $60 \%$ and $40 \%$ Span

Figure 7. Cross-Spectral Density Functions Between Differential Pressures at Stations on Flexible Tail, 16\% F/A-18 Model, 34 Degrees AOA

The purpose of this paper is to illustrate that the spatial correlation of the buffet pressures on an F/A-18 vertical tail in flight agrees with the partial correlation observed on a $16 \%$ F/A-18 vertical tail in the Transonic Dynamics Tunnel at the NASA Langley Research Center.

\section{$\underline{\text { Some Flight Test Results }}$}

Pressures on the surfaces of the starboard vertical tail, shown in Figure 8, of the High Alpha Research Vehicle (HARV), shown in Figure 1, were measured at various flight conditions using a sampling rate of $320 \mathrm{~Hz}$. Cross-spectral density functions of the unsteady differential pressures at Mach 0.3 and 30 degrees angle of attack were computed from the digitized time histories of 43 seconds in length using a block size of 2048 with $75 \%$ overlapping and a rectangular window.

The magnitude and phase of the cross-spectral density (CSD) functions, plotted in Figure 9, illustrate the spatial features of the differential pressures along the $85 \%$ span line. Based on the location of the peak magnitude of the CSD functions in Figures 9.a, 9.b and 9.c, the dominant frequency component of the wave form is approximately $11.25 \mathrm{~Hz}$. This consistency indicates that similar differential pressure waves are observed at all stations along the $85 \%$ span line. 


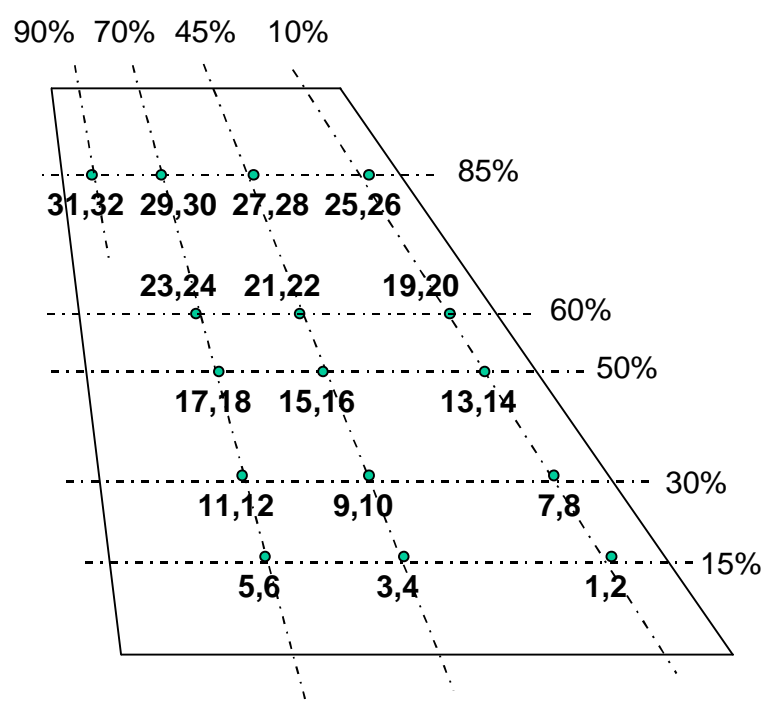

Figure 8. Location of Pressure Transducer on Starboard Vertical Tail, High Alpha Research Vehicle (HARV)

The value of the phase at the dominant frequency of $11.25 \mathrm{~Hz}$ is different for the CSD functions, as shown in Figure 9. For instance, in Figure 9.a, the phase at $11.25 \mathrm{~Hz}$ is approximately 27 degrees while, in Figure 9.b, the phase at $11.25 \mathrm{~Hz}$ is approximately 45 degrees.

Cross Spec: (KP25- KS26) / (KP27- KS28)
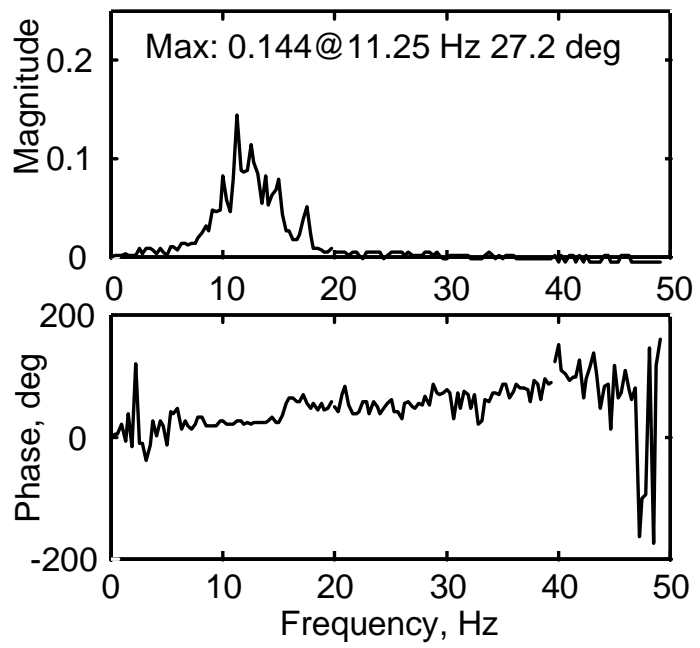

Figure 9.a. Cross-Spectral Density Function Between Differential Pressures on HARV, Station KP25-KS26 With Respect to KP27-KS28, Mach 0.3, 30 Degrees AOA, LEX Fence Off
Cross Spec: (KP25- KS26) / (KP29- KS30)
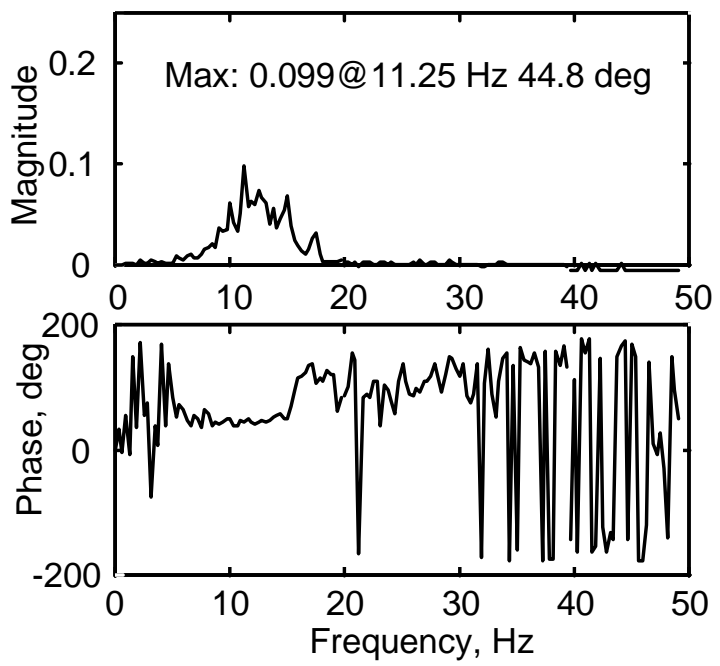

Figure 9.b. Cross-Spectral Density Function Between Differential Pressures on HARV, Station KP25-KS26 With Respect to KP29-KS30, Mach 0.3, 30 Degrees AOA, LEX Fence Off

Cross Spec: (KP25- KS26) / (KP31- KS32)
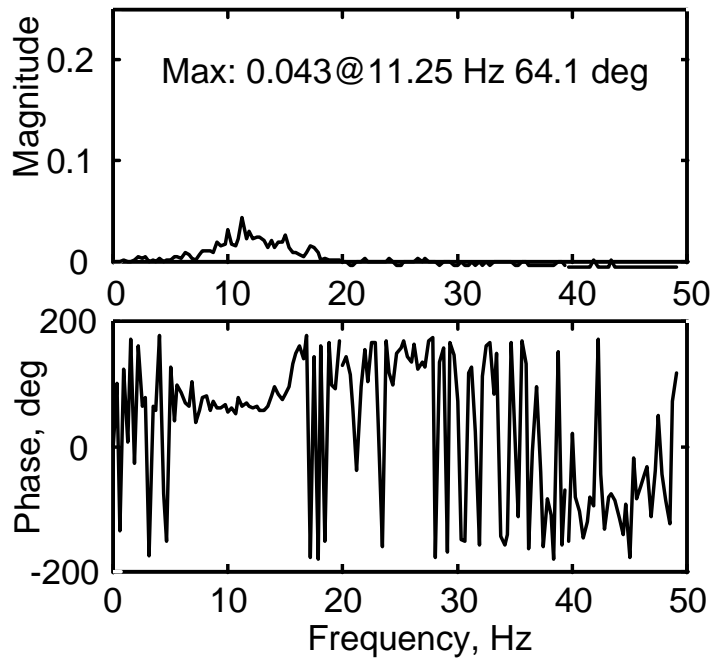

Figure 9.c. Cross-Spectral Density Function Between Differential Pressures on HARV, Station KP25-KS26 With Respect to KP31-KS32, Mach 0.3, 30 Degrees AOA, LEX Fence Off

The variation of phase is dependent on the distance between differential pressure stations along the $85 \%$ span line. The pressure wave, represented by the CSD in Figure 9.a, traveled $35 \%$ of the chord length along the $85 \%$ span line 
while the pressure wave, represented by the CSD in Figure 9.b, traveled $60 \%$ of the chord length along the 85\% span line, as shown in Figure 8.

As a check, the phase, shown in Figure 9.a, is computed for the pair of differential pressure transducers KP25-KS26 and KP27-KS28 which are 1.54 feet apart. At 30 degrees AOA, the velocity of the stream lines in the vicinity of the tail will be less than the flight speed of Mach 0.3 (330 feet per second). From Reference 12, the velocity near the tail will be approximately $70 \%$ of the free stream value. Therefore, using Equation 1, where " $\mathrm{f}$ " is the frequency of interest (11.25 Hz in this case), "d" is the distance between stations, and " $U$ " is the velocity of the streamline near the $85 \%$ span line, the phase at $11.25 \mathrm{~Hz}$ is computed as 27 degrees, which agrees well with the value shown in Figure 9.a. Similarly, the phase may be computed for other pressure transducer pairs.

$$
\phi=\omega \cdot t=2 \pi f \cdot \frac{d}{U} \cdot \frac{180}{\pi}
$$

Equation 1 may be simplified further by introducing the Strouhal number, defined by Equation 2, and canceling like terms, to yield Equation 3.

$$
\begin{aligned}
& \mathrm{n}=\frac{\mathrm{fd}}{\mathrm{U}} \\
& \phi=360 \mathrm{n}
\end{aligned}
$$

Since dispersion (break down of eddies into higher harmonics) and dissipation (energy loss) are expected in this highly turbulent flow near the tail, the pressure wave is expected to deform as it travels along the tail ${ }^{12}$. Therefore, the magnitude of the partial correlation of the differential pressures at two stations is expected to drop as the distance between these two pressure stations increases. The coherence function provides a tool for assessing this drop in correlation. In Figure 10 , coherence functions are computed, with respect to KP25-KS26, for the differential pressures at selected stations for Mach 0.3, 30 degrees AOA.

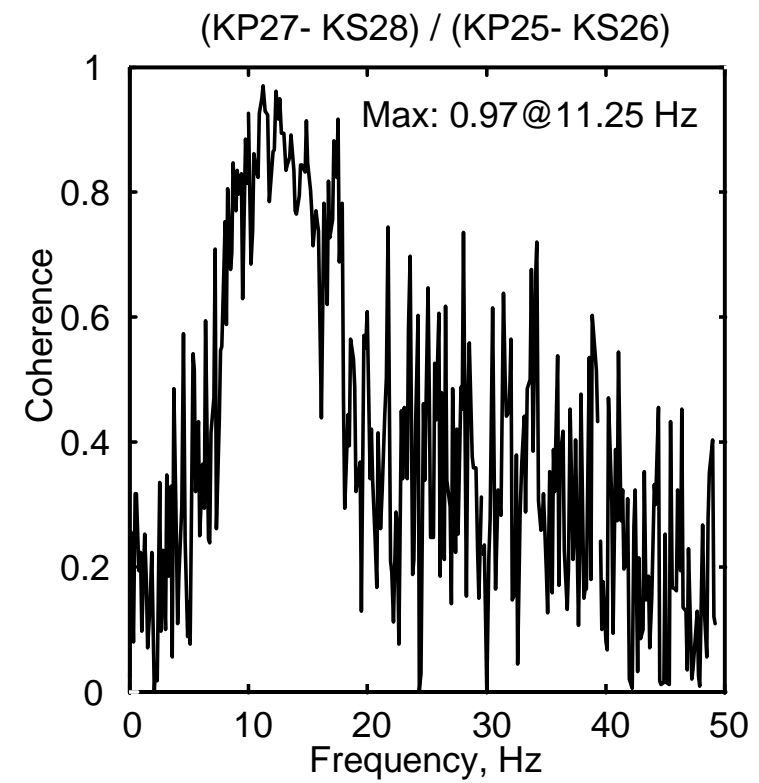

Figure 10.a. Coherence Function Between Differential Pressures on HARV, Station KP27-KS28 With Respect to KP25-KS26, Mach 0.3, 30 Degrees AOA, LEX Fence Off

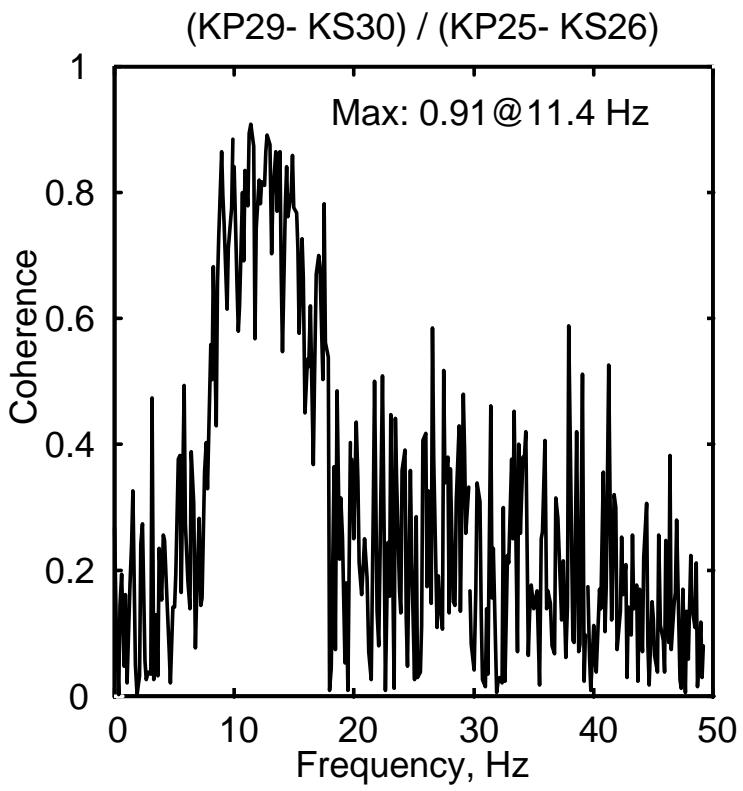

Figure 10.b. Coherence Function Between Differential Pressures on HARV, Station KP29-KS30 With Respect to KP25-KS26, Mach 0.3, 30 Degrees AOA, LEX Fence Off 


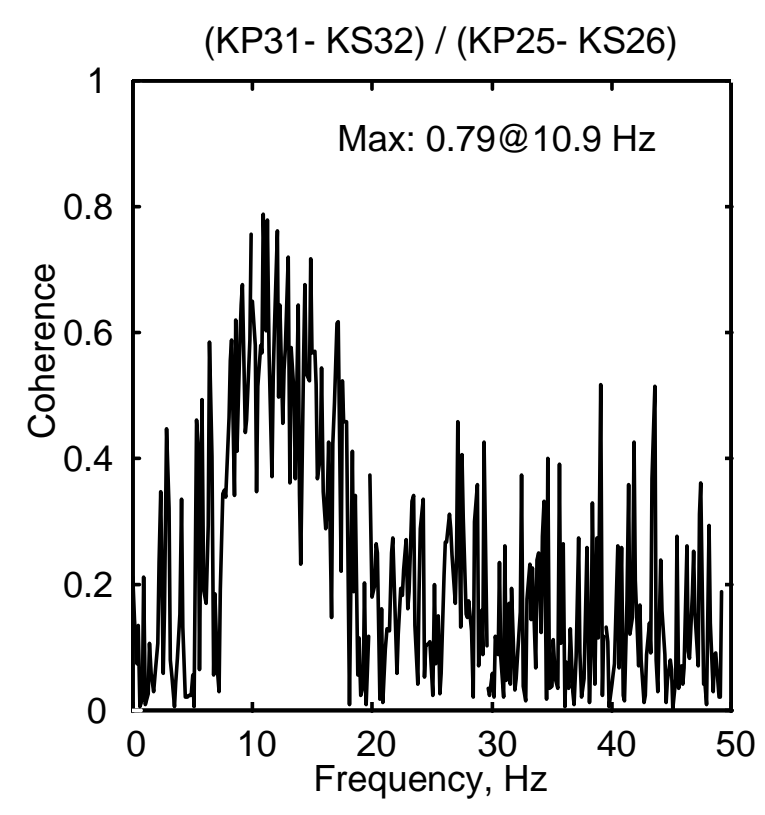

Figure 10.c. Coherence Function Between

Differential Pressures on HARV,

Station KP31-KS32 With Respect to KP25-KS26,

Mach 0.3, 30 Degrees AOA, LEX Fence Off

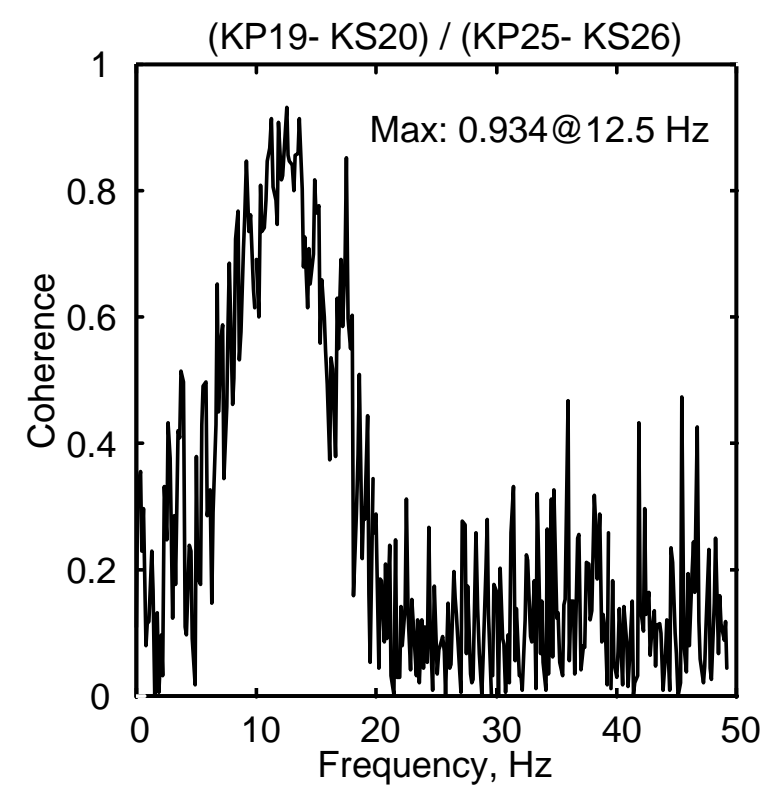

Figure 10.d. Coherence Function Between Differential Pressures on HARV,

Station KP19-KS20 With Respect to KP25-KS26, Mach 0.3, 30 Degrees AOA, LEX Fence Off

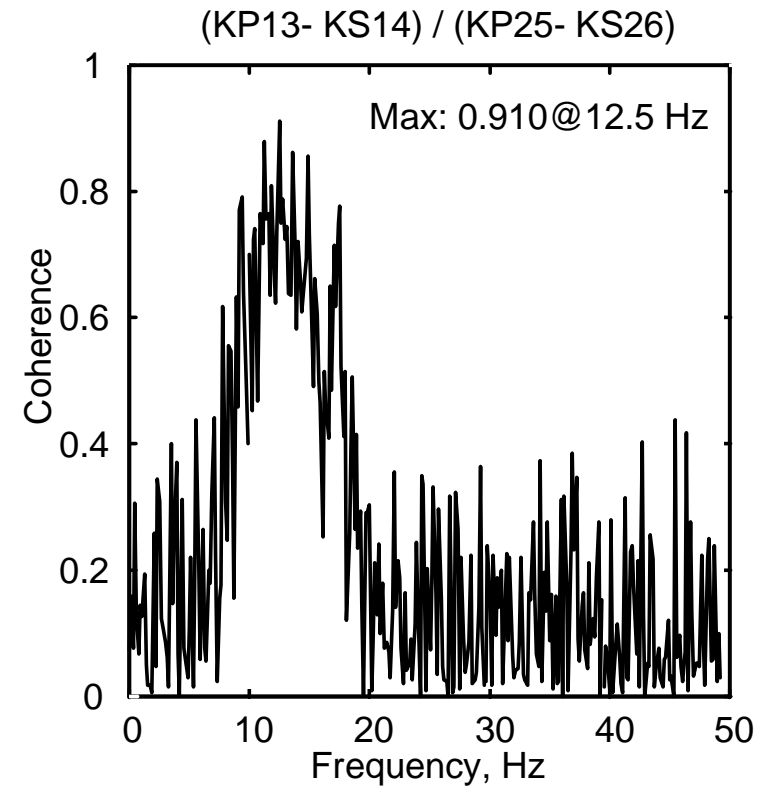

Figure 10.e. Coherence Function Between Differential Pressures on HARV, Station KP13-KS14 With Respect to KP25-KS26, Mach 0.3, 30 Degrees AOA, LEX Fence Off

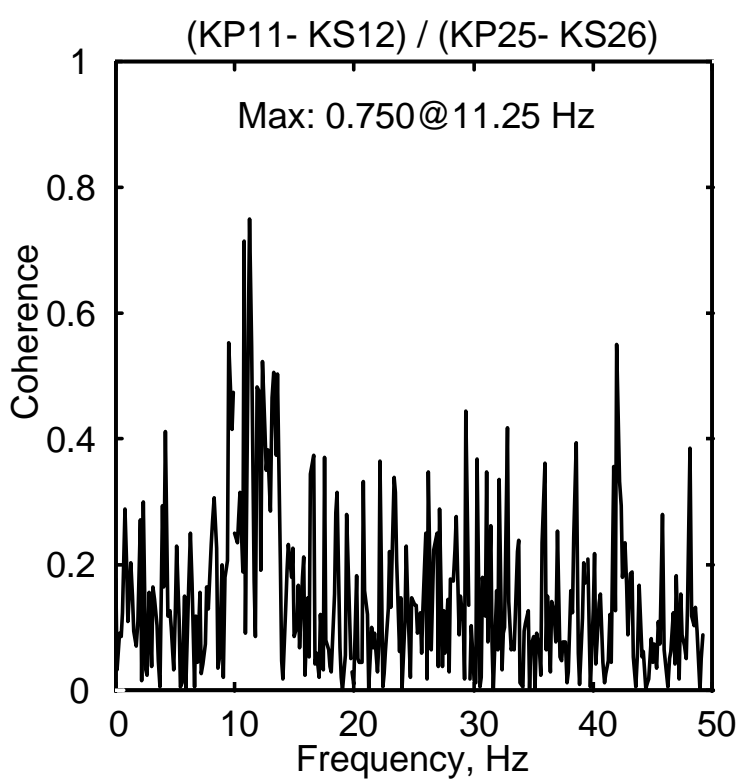

Figure 10.f. Coherence Function Between Differential Pressures on HARV, Station KP11-KS12 With Respect to KP25-KS26, Mach 0.3, 30 Degrees AOA, LEX Fence Off 
In Figure 10.a, the maximum value of the coherence is 0.97 (out of a possible 1.0) at 11.25 $\mathrm{Hz}$, the dominant frequency in the pressure wave form. The values of the coherence for other frequency components in the pressure wave are less than 0.97. This feature indicates that some dispersion of the frequency components is occurring as the wave moves along the tail, but the dominant frequency component is dispersing only slightly. For instance, if the values of the coherence for all frequency components are unity, then no dispersion occurs in the wave as it moves along the tail. Conversely, if the values of the coherence for all frequency components gets smaller as the distance between stations increases, then all frequency components in the wave are dispersing as a function of distance. The latter case is the nature of the unsteady differential pressures that occur on the vertical tails during buffet caused by LEX vortex burst.

As the pressure wave moves aft along the $85 \%$ span line, the maximum value in the coherence function falls from 0.97, shown in Figure 10.a, to 0.91, shown in Figure 10.b., to 0.79, shown in Figure 10.c. Therefore, some dispersion and possibly some dissipation are occurring along a constant span line. For a constant chord line, the maximum values in the coherence functions are near unity. For the $10 \%$ chord line, the maximum value in the coherence functions are 0.934 , shown in Figure 10.d, and 0.91, shown in Figure 10.e. The smallest maximum values of the coherence functions occur for stations that are farthest apart, as seen in Figure 10.f, where the maximum value is 0.75 .

\section{Comparison of Flight Results With Wind-Tunnel Results}

The CSD functions, shown in Figure 7, that were computed for pressures measured in the Transonic Dynamics Tunnel on a 16\% F/A-18 vertical tail at Mach 0.1 (110 feet per second), are representative of an F/A-18 at Mach 0.6 (660 feet per second) at 34 degrees AOA. Based on the Strouhal number, shown in Equation 2, a doubling of flight speed will reduce the phase shift by $50 \%$, and as shown in Reference 1, a doubling of the flight speed will also double the frequency value at which the peak magnitude of the CSD occurs. Therefore, a direct comparison of the phase value at the peak magnitude of the CSD function is possible between wind-tunnel results for the $16 \%$ F/A-18 model and the results of the HARV at Mach 0.3.

In Figure 11, the CSD plot shows that the phase between the differential pressure at Station 3 with respect to Station 1 on the $16 \%$ F/A-18 vertical tail, shown in Figure 5, at 34 degrees angle of attack is approximately 46 degrees. For similar conditions and stations on the HARV, the phase is approximately 45 degrees, as shown in Figure 9.b.

Diff $\mathrm{P}$ at Station 3 / Diff $\mathrm{P}$ at Station 1

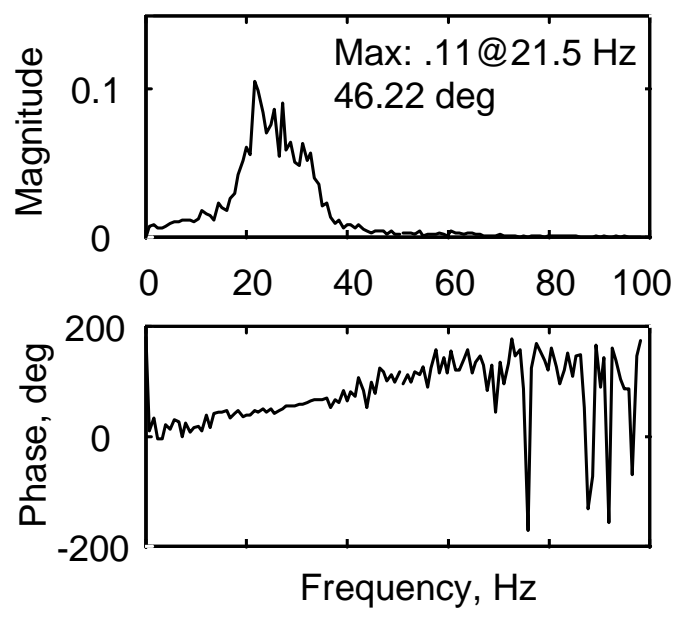

Figure 11. Cross-Spectral Density Function Between Differential Pressures on 16\% F/A-18

Model, Station 3 With Respect to Station 1, Mach 0.6 (Simulated), 34 Degrees AOA

Coherence functions were computed for selected stations on the $16 \%$ F/A-18 flexible vertical tail for comparing coherence functions computed for the HARV. Shown in Figure 12.a, the maximum value of the coherence function between stations 4 and 5 on the $16 \%$ F/A-18 vertical tail, shown in Figure 5, is 0.844. This value, shown in Figure 12.a, lies between the maximum values of the coherence functions, shown in Figures 10.b and 10.c, for similar stations on the HARV vertical tail, shown in Figure 8. For an additional comparison, the coherence function between two 
of the most separated stations on the $16 \%$ F/A-18 flexible vertical tail was computed, as shown in Figure 12.b. The maximum value of the coherence between stations 3 and 13 on the $16 \%$ F/A-18 vertical tail, shown in Figure5, is 0.625. This value, as shown in Figure 12.b, is less than the maximum value of 0.75 for the coherence function, shown in Figure 10.f, between stations that are similarly separated in terms of percent chord and percent span.

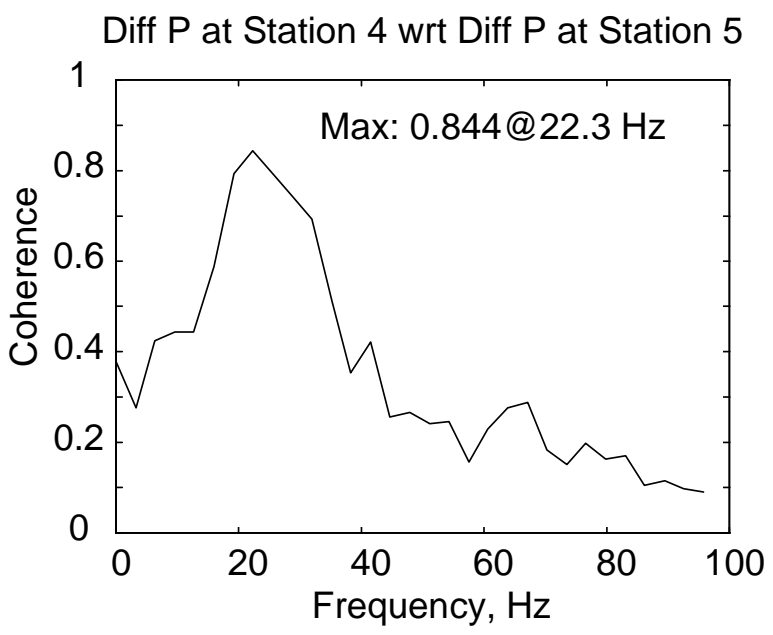

Figure 12.a. Coherence Function Between Differential Pressures on 16\% F/A-18 Model,

Station 4 With Respect to Station 5, Mach 0.6 (Simulated), 34 Degrees AOA

Diff $P$ at Station 3 wrt Diff $P$ at Station 13

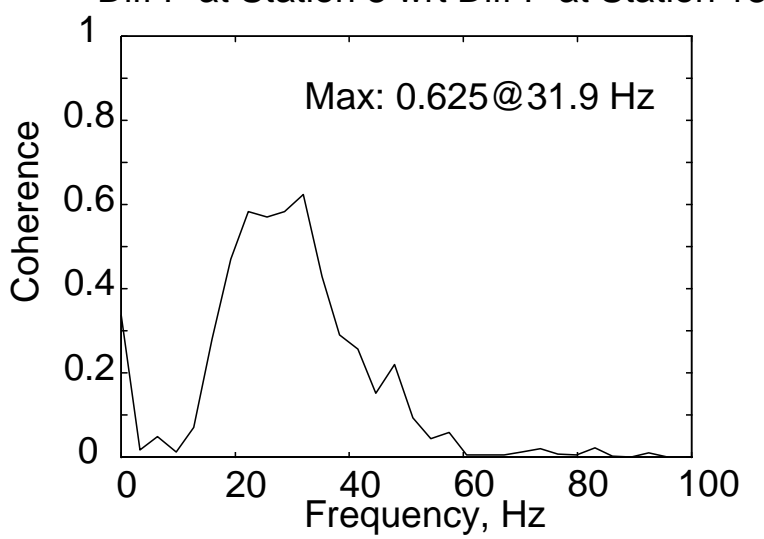

Figure 12.b. Coherence Function Between

Differential Pressures on 16\% F/A-18 Model,

Station 3 With Respect to Station 13, Mach 0.6 (Simulated), 34 Degrees AOA

\section{$\underline{\text { Conclusions }}$}

Cross-spectral density and coherence functions were presented for indicating the pressure correlation that occurs on the vertical tail of the F/A-18 configuration during LEX vortex burst. The unsteady buffet pressures that are caused by LEX vortex burst during high angle of attack maneuvers on the F/A-18 (HARV) are not fully correlated as previously assumed. In fact, the local Strouhal number may be used to relate the phase shift of the unsteady buffet pressures between stations on the vertical tail. The magnitude of the cross-spectral density functions presented herein indicate that the vortex disturbance reduces as it travels along the tail. In agreement with this finding, the magnitude of the coherence functions illustrate that the correlation between unsteady pressures at different stations is affected by the separation distance between the stations. One issue that surfaces from this finding is the role, if any, that the vertical tail plays in affecting the spatial correlation and dispersion of the unsteady buffet pressures.

As shown herein, the results presented for the HARV agree well with the partial correlation of the buffet differential pressures measured on a 16\% F/A-18 model in the Transonic Dynamics Tunnel. Through referenced previous comparisons with other wind-tunnel model data, the 16\% F/A-18 data, and thus the F/A-18 (HARV) data, consistently illustrate the partial correlation of the differential pressures that occur on the vertical tail during LEX vortex burst at high angles of attack.

\section{Acknowledgments}

The authors would like to extend their gratitude to the test crews that conducted the investigations and reduced the pressure data to a useable format: NASA Dryden Flight Center, NASA Langley Research Center, and Wright Laboratory. In addition, a special thanks is extended to Ms. Sheri Hoadley and Ms. Carol Wieseman for the software used to compute and plot the CSD, crosscorrelation, and coherence functions. 


\section{$\underline{\text { References }}$}

1 Zimmerman, N. H., and Ferman, M. A., "Prediction of Tail Buffet Loads for Design Application," Vols. I and II, Rept. No. NADC-88043-60, July 1987.

2 Lee, B. H. K., Brown, D., Zgela, M., and Poirel, D., "Wind Tunnel Investigation and Flight Tests of Tail Buffet on the CF-18 Aircraft", in Aircraft Dynamic Loads Due to Flow Separation, AGARD-CP-483, NATO

Advisory Group for Aerospace Research and Development, Sorrento, Italy, April 1990.

3 Lee, B.H.K. and Tang, F. C., "Unsteady Pressure and Load Measurements on an F/A18 Vertical Fin at High-Angles-of-Attack," AIAA-92-2675-CP, $10^{\text {th }}$ Applied Aerodynamics Conference, Palo Alto, CA, June 22-24, 1992.

4 James, K. D. and Meyn, L. A., "Dependence on Integrated Vertical-Tail Buffet Loads For F/A-18 on Sensor Density," SAE Technical Paper 94110, Aerospace Atlantic Conference and Exposition, Dayton, Ohio, April 18-22, 1994.

5 Bean, D. E. and Lee, B. H. K., "Correlation of Wind Tunnel and Flight Test Data For F/A-18 Vertical Tail Buffet," AIAA-94-1800-CP, $12^{\text {th }}$ AIAA Applied Aerodynamics Conference, Colorado Springs, CO, June 20-22, 1994.

6 Pettit, C. L., Banford, M., Brown, D., and Pendleton, E., "Pressure Measurements on an F/A-18 Twin Vertical Tail in Buffeting Flow," Vols 1-4, United States Air Force, Wright Lab., TM-94-3039, Wright Patterson AFB, OH, August 1994.

7 Meyn, L. A. and James, K. D., "Full-Scale Wind-Tunnel Studies of F/A-18 Tail Buffet," Journal of Aircraft, Vol. 33, No. 3, May-June 1996.

8 Moses, R. W., "Active Vertical Tail Buffeting Alleviation on a Twin-Tail Fighter Configuration In a Wind Tunnel," presented at the CEAS International Forum on Aeroelasticity and Structural Dynamics 1997, 17-20 June 1997, Rome, Italy.

9 Bendat, J. S. and Piersol, A. G., Engineering Applications of Correlation and Spectral
Analysis, Second Edition, John Wiley \& Sons, Inc., 1993.

10 Moses, R. W. and Pendleton, E., “A Comparison of Pressure Measurements Between a Full-Scale and a 1/6-Scale F/A-18 Twin Tail During Buffet," presented at the 83rd Meeting of the Structures And Materials Panel (SMP) of the Advisory Group for Aerospace Research and Development (AGARD), Florence, Italy, September 2-6, 1996.

11 Moses, R. W. and Ashley, H., "Spatial Characteristics of the Unsteady Differential Pressures on $16 \%$ F/A-18 Vertical Tails," $36^{\text {th }}$ AIAA Aerospace Sciences Meeting and Exhibit, Reno, Nevada, January 12-15, 1998.

12 Moses, Robert W., "Spatial Characteristics of the Unsteady Differential Pressures on Vertical Tails of a Twin-Tailed Aircraft at High Angles of Attack With an Emphasis on Buffeting Alleviation," Ph.D. Dissertation, Stanford University, August 1997. 